\title{
Charge fluctuations in coupled systems: ring coupled to a wire or ring
}

\author{
P. Singha Deo ${ }^{1}$, P. Koskinen ${ }^{2}$, and M. Manninen ${ }^{2}$ \\ 1 Unit for Nano-science \& Technology, S. N. Bose National Centre for Basic Sciences, \\ JD Block, Sector III, Salt Lake City, Kolkata 98, India. \\ ${ }^{2}$ Nanoscience Center, Department of Physics, University of Jyvaskyla, PO Box - 35, 40014 Jyvaskyla, Finland.
}

(Dated: November 20, 2018)

\begin{abstract}
Coupled systems in mesoscopic regime are of interest as charge fluctuation between the subsystems will depend on electron-electron interactions and will play a dominant role in determining their thermodynamic properties. We study some simple systems like a stub or a bubble strongly coupled to a ring. We show that for strong electron-electron interaction, there are some regimes where these charge fluctuations are quenched and charge is individually conserved in the two subsystems. This feature does not depend on choice of parameters or charge distribution.
\end{abstract}

PACS numbers:

\section{INTRODUCTION}

Coupled mesoscopic samples are very challenging to understand. Transfer of charge and charge fluctuations between different parts of a coupled system, can dramatically alter its properties $[1,2]$. Electron-electron interactions strongly dictate such charge transfer and fluctuations.

If one starts with a system that has translational invariance or rotational invariance then electron-electron interactions preserve the invariance. This often helps in solving an interacting electron system. However, if we start with a system that has no translational or rotational invariance, the the problem of solving the interacting electrons in the system is non-trivial. Coupled systems do not have rotational or translational invariance and this makes the problem very difficult.

In this regard, simple systems like finite one dimensional (1D) systems, are what researchers intend to understand first $[1,2]$. With the advent of mesoscopic systems, one can now realize such finite and $1 \mathrm{D}$ systems in the laboratory and this has further encouraged scientists to examine the role of interactions in such simple systems $[1,2]$ as that considered in Fig. 1 (a). It consists of a 1D ring connected to a $1 \mathrm{D}$ finite wire (referred to as stub). In Fig. 1 (b) we replace the stub by a small ring that we refer to as bubble. An Aharonov-Bohm flux through the center of the ring induces a persistent current in the ring [3], due to which the system has a net magnetization. There is no flux through the bubble. The persistent current in the $n$th energy level is defined as $I_{n}=-c \frac{\partial E_{n}}{\partial \phi}$, where $E_{n}$ is the $n$th energy eigenvalue, $c$ is the velocity of light and $\phi$ is the flux through the ring. States in the ring are plane wave like extended states that carry current (due to the flux piercing it), while the states in the stub or in the bubble are like the localized states of a cavity. These systems have been studied earlier by various authors. The parity effect was studied in $[4,5]$, persistent currents in such a system in the diffusive regime was studied in ref. [6], conductance across such a system [7] and persistent currents in such a system in the ballistic regime [8] has also been studied.
Buttiker and Stafford [1] considers the stub to be weakly coupled to the ring. In this weak coupling limit they consider the regime wherein in the non-interacting limit an empty stub state (ESS) weakly hybridizes with an occupied ring state (ORS) and the Fermi energy coincides with the hybridized states. In this regime an effective theory was put forward to account for electronelectron interactions. The effective theory ignored the presence of all other states apart from the ESS and ORS and included electron interactions with the help of a geometrical capacitance. On the other hand, Cho et al [2], consider again the weakly coupled regime, when the stub state is below the Fermi energy, in the Kondo regime. Electron-electron interactions was treated in the infinite $U$ limit of the Hubbard model, using the slave boson representation.

\section{THE PROBLEM}

On one hand, the quantum mechanical uncertainty tends to create charge fluctuations in the system and on the other hand the electron-electron interactions tend to localize the electrons and destroy the charge fluctuations. Situations, wherein charge fluctuations are completely quenched are of fundamental importance in physics. Several novel effects like quantum phase transitions, deformations of nuclei [9], deformations of electronic states in quantum dots [10] and clusters [11], fractional periodicity of persistent currents [12] are all related to quenching of charge fluctuations. So such a competition between localization and delocalization may also play some important role in the case of charge fluctuations in coupled mesoscopic systems and this is what we intend to investigate in this paper.

In this paper we consider the regime of strong coupling between the sub-systems for any arbitrary interaction strength. In the strong coupling regime, it is not possible to ignore states other than ESS and ORS. Also it is not possible to treat electron-electron interactions in the form of a geometrical capacitance or the slave boson formalism. We solve the problem numerically, mak- 
ing exact diagonalization of Hubbard type Hamiltonian. We show that at certain Fermi energies, and at certain regimes which we call the Fano regime, the electronic states of the strongly interacting system can be predicted by looking at the non-interacting single particle levels. Charge fluctuations between the ring and the stub can be easily quenched in these special regimes by electron interactions and this is the main reason for such behavior. There is a non-symmetry dictated node (NSDN) in the many body wave function in this Fano regime that is responsible for making charge conserved and integral in the two sub-systems and hence such a behavior. In other regimes such a simple picture cannot be visualized.

\section{THE MODEL}

Schematic figures of the geometries considered are shown in Fig. 1 (a) and (b). The systems are made up of sites (shown as bold dots) to be described by the Generalized Hubbard Model. The sites in the stub or in the bubble are labeled as A and B. Due to the presence of the stub or bubble, the system has no rotational symmetry. We use the generalized Hubbard model to find the electronic states of the system. The generalized Hubbard Hamiltonian is

$$
\begin{aligned}
H= & \sum_{i, \sigma} \epsilon_{i} c_{i, \sigma}^{\dagger} c_{i, \sigma}+\left(t e^{i \alpha / R} \sum_{<i j>, \sigma} c_{i, \sigma}^{\dagger} c_{j, \sigma}+H C\right) \\
& +U \sum_{i, \sigma \neq \sigma^{\prime}} n_{i, \sigma} n_{i, \sigma^{\prime}}+V \sum_{<i j>, \sigma} n_{i, \sigma} n_{j, \sigma^{\prime}}
\end{aligned}
$$

Here $n_{i}=c_{i}^{\dagger} c_{i}, c_{i}$ is electron annihilation operator at site i, $\sigma$ is spin index, and $\epsilon_{i}, t, U, V$ are parameters that have their usual meanings: on-site energy, hopping integral, on-site Coulomb interaction and nearest neighbor Coulomb interaction, respectively. $R$ is the total number of sites in the ring only, $\alpha=2 \pi \phi / \phi_{0}, \phi_{0}=h c / e$ and $\phi$ is the flux through the ring. The number of sites making the stub or bubble is denoted as $\mathrm{S}$.

\section{THE SINGLE PARTICLE STATES}

In this section we shall briefly review what is known about the single particle states of coupled systems. In Fig. 2. we show the single particle levels for $\mathrm{U}=0$ and $\mathrm{V}=0$ for a system as in Fig. 1 (a), with 9 sites in the ring and 2 sites in the stub. Thus this is a non-interacting system and the first 6 single particle levels as a function of flux are shown in Fig. 2. The ground state is diamagnetic as it has a minimum at zero flux and energy increases with flux. The first excited state is paramagnetic as it has a maximum at zero flux and energy decreases with flux. All the 6 energy levels have a magnetization associated with it except the 4 th. One can see that the 4 th level from below (i.e., the 3 rd excited state) is independent of flux. This is due to the NSDN [13]. There is a node in the wave-function of the $3 r d$ excited state at the junction between the ring and the stub. We refer to this node as non-symmetry dictated node, a term originally coined by Leggett [14]. Due to this node, the wave-function in the 3rd excited state do not see the magnetic flux. Note that the energy of this state is $E=-E_{0}$. There can be several symmetry dictated nodes (like nodes due to box quantization or nodes due to antisymmetry property of many body wave-function) that do not make a state flux independent. The NSDN is forced by the boundary condition at the free end of the stub. If we cut the ring at one point and attach leads to the two broken ends then we get the system shown in Fig. 3 (a) and (b). The transmission through this system at an energy $E=-E_{0}$ show an absolute 0 due to this NSDN. Such a resonance is called Fano resonance [15]. It is easy to find situations wherein such flux independent states occur as it can be determined from diagonalization of the non-interacting Hamiltonian. Alternately, it can be found even more easily, by inspecting the numbers of sites and this follows from a general theory of parity effect [13]. For example it happens for a $(6+2)$ system, $(12+2)$ system, $(15+2)$ system, $(9+3)$ system and so on. By $(6+2)$ system we mean 6 sites in the ring and 2 sites in the stub and so on. A $(9+2)$ system will have eleven levels that will change slope 9 times as there are 9 sites in the ring. The ground state will be diamagnetic. The first excited state will change slope for the first time to be paramagnetic. To change the slope 9 times, the 4 th and 8 th state has to be flux independent or else the particle hole symmetry will be broken. Even if we place the two sites of the stub as in Fig 1 (b) so that the two sites make a bubble, even then the 3rd excited state is independent of flux. We shall show that the strongly interacting system in situations where single particle levels show flux independence has some general features that do not depend on parameters of the system.

\section{THE MANY BODY STATES}

We take the same system as that in Fig. 2, i.e., a ringstub system with $S=2$ and $R=9$, and put 4 spin up and 4 spin down electrons in it. In the non-interacting limit, where each single particle level is 2 fold spin degenerate, the Fermi energy will coincide with the 4th level which is flux independent. The 4 th level will accommodate 1 spin up electron and 1 spin down electron and the persistent current or magnetization of the system will depend on 3 up and 3 down electrons in the first 3 energy levels. A small amount of electron-electron interaction or disorder in site energies will perturb the total energy of the system and one cannot think of any particle not affected by flux. In Fig. 4 we plot the occupation probability $\left\langle n_{A}\right\rangle$ of site $\mathrm{A}$ and $\left\langle n_{B}>\right.$ of site $\mathrm{B}$ in the presence of a small amount of interaction. Here $\left\langle n_{A}\right\rangle$ means the expectation value of $c_{A}^{\dagger} c_{A}$ and so on. We have considered $\left\langle n_{A}\right\rangle$ 
and $<n_{B}>$ for spin up electrons only as that for spin down is identically the same due to spin degeneracy in absence of an explicit magnetic field. One can see that the total occupation probability of the two sites in the stub, i.e., the sum of $\left\langle n_{A}\right\rangle$ and $\left\langle n_{B}\right\rangle$ is strongly flux dependent. It means that Aharonov-Bohm flux can cause charge transfer and charge fluctuation between the stub and the ring. This is a gauge dependent charge transfer in a system at equilibrium as opposed to non-equilibrium bias created charge transfer. Normally in quantum dots, one uses the bias induced charge transfer, wherein a small voltage bias is applied to pump a charge into the quantum dot. In a closed coherent system as that considered in this work, changes in equilibrium parameters in the Hamiltonian can lead to such charge transfers. Such charge transfers can drastically alter the thermodynamic properties of the sample like in this case the magnetization of the sample. Hence it is important to understand these charge transfers. Also it is important to understand situations wherein charge remains conserved, does not fluctuate, and what kind of conservation it is. For example we have seen that in the non-interacting limit, the 4th spin up and spin down electrons remain completely conserved in the stub in a flux independent state. This happens because of the NSDN (non-symmetry dictated node) in the non-interacting wave function. But once there are interactions, the non-interacting wave functions are perturbed. Then the NSDN has little or no role to play and everything is dominated by interactions.

However, if we make $U$ and $V$ much larger, then there is the same amount of integer charge localized in the stub as in the non-interacting case. This can be seen in Fig. 5 . This is counter-intuitive. Because as we increase the repulsive interaction between electrons, we will expect that the charge in the two neighboring sites in the stub will decrease as long as total charge concentration in the ring is lower than in the stub. But instead the stub sites acquire more and more charge in order to make the total charge in the stub same as that in the non-interacting case and that is an integer. Here if we take the sum of $\left\langle n_{A}\right\rangle$ and $\left\langle n_{B}\right\rangle$, then the sum is constant in flux and close to 1 as shown by the dotted line in Fig. 5. They can individually change by appreciable amounts, implying that we have not yet reached the localized regime wherein each electron gets localized into a crystal [12]. Charge can fluctuate between sites $A$ and $B$ and so can charge fluctuate between different sites in the ring to carry an appreciable amount of persistent current. But total persistent current is like that of 3 spin up and 3 spin down electrons in a ring. This will be illustrated in detail below.

We want to stress that this is a general feature and it happens for all system sizes provided it is in the Fano regime, which means that in the non-interacting limit there are flux independent states that coincide with the Fermi energy. Even if we put some disorder in the site energies, we get the same result as in Fig. 5, as long as the site energies are much smaller than the strength of electron-electron interactions. For example if we take a $6+2$ system then in the non-interacting limit, the $3 \mathrm{rd}$ single particle state is flux independent. So, if we put 3 spin up and 3 spin down electrons in the system, then in the strongly interacting limit, 1 up and 1 down electron remain completely localized in the stub and the magnetization of the system is determined by 2 up and 2 down electrons in the ring. In Fig. 6, we have taken a $6+2$ system with 3 up and 3 down electrons in it. We have also taken the strong interaction limit, when 1 up and 1 down electrons are localized in the stub. We have plotted the first 6 many body energy levels of the system that exhibit large gaps at the zone boundaries $\alpha=0,0.5,1$ and level crossings within the zone. Level crossings within the zone is a precursor of Wigner crystallization [12] and is not related to the breakdown of rotational symmetry. The gaps at the zone boundary are due to the absence of rotational symmetry. In Fig. 7, we have taken just a ring of 6 sites with a defect, and plotted the first 6 energy levels as a function of $\alpha$ when there are 2 up and 2 down electrons in the ring, in the same strong interaction limit as in Fig. 6. The energy levels are very similar to that in Fig. 6, which implies that in Fig. 6, the persistent current is completely determined by 2 up and 2 down electrons in the ring. Charge localized in the stub does not contribute to persistent current and also charge fluctuations inside the stub has no qualitative effect on the persistent current in the ring. There can be non-interacting situations [17] wherein charge fluctuations do not influence propagating states and in our system this seems to happen for strong interactions in the Fano regime.

Note that when the stub is made of only 2 sites, then in strong interaction limit, accommodating 1 up and 1 down electrons is almost pushing the stub to its maximum capacity. If we make the stub having more sites, then it happens more easily and with smaller values of $U$ and $V$. For example, if we take a $8+3$ system, then in the noninteracting limit, we find that the $3 \mathrm{rd}$ and the 6 th single particle levels are flux independent. If we put 3 up and 3 down electron in the system, then in the non-interacting limit as well as in the strong interacting limit, 1 up and 1 down electrons are localized in the stub. If we put 6 up electrons and 6 down electrons in the system, then again in both limits, 2 up and 2 down electrons are localized in the stub. In all these different systems, the ratio of charge in the stub to the charge in the ring is different. But, nevertheless, quite generally an integer amount of charge gets localized in the stub. If we go away from the Fano regime, then it is not possible to have an integer amount of charge in the stub and hence the behavior of the system cannot be understood in terms of the levels of the ring or in terms of the levels of the stub.

It can be seen that in the strong interaction limit, in the presence of NSDN, the charge density in the stub can be much higher (or lower) than the charge density in the ring. Charge in the stub increases as we increase the repulsive interaction between the electrons and saturates when the total charge in the stub becomes an integral number same as that in the non-interacting limit. This is 
as if there is an effective attraction between the electrons in the stub. One may think that this effect is because the stub sites have a different coordination number than the ring sites. For example site A in Fig. 1 (a) has only one nearest neighbor while all sites in the ring has at least two nearest neighbors. However, this effect happens for all possible parameters that gives different ratio of total charge in the stub to the total charge in the ring. Also, in Fig 1 (b) the sites in the bubble are equivalent to the sites in the ring in regard to coordination number. If we do similar calculation for Fig. 1 (b) as we did in Fig. 5, then we expect $\left\langle n_{A}\right\rangle=\left\langle n_{B}>\right.$ due to the symmetry of the two sites $A$ and $B$. We also find that that for the same interaction strength and same number of ring sites as that in Fig. $5,<n_{A}>=<n_{B}>=0.491$, which is very close to the average of the two solid lines in Fig. 5. This strongly suggests that coordination number is not important. It is the non-symmetry dictated node that makes the total charge in the stub or bubble similar to that of the non-interacting system. As a result the total charge in the stub or bubble tends to be an integer in spite of strong repulsion. The combined effect of a NSDN and strong repulsive interaction is like an effective electron-electron attraction in the stub or in the bubble.

The clean interacting ring or a ring with a defect is well understood in terms of an effective Hamiltonian [16], because of which we can understand the magnetization of a ring of any arbitrary number of sites and arbitrary number of electrons. In our case, after electrons are localized in the stub, the stub behaves like a static impurity. This gives us an easy way of understanding ring stub systems that are beyond the reach of exact diagonalization methods. When applying approximate methods, this can provide a simple guideline for cross checking results in some limits like the Fano limit.

Quantum mechanical study of coupled atoms date back to 1923 [18]. Analogous study has also been made using quantum dots [19]. The starting point is well separated quantum dots or two dots with a large electrostatic bar- rier in between. Then one gradually enhance the coupling between the dots by decreasing the barrier or by bringing the dots close to each other. Thus charge in each dot can be controlled and made integer by physically decoupling the two dots. The study of coupled geometries as presented in this paper also seems to be an avenue for searching new phenomenon and physics. We show that we can have integral charge in the two sub-systems not by physically decoupling them but due to an internal mechanism.

\section{CONCLUSIONS}

The non-interacting wave function in coupled systems can have NSDN. Interactions perturb the non-interacting wave function and the NSDN is destroyed. But in the limit of strong electron-electron interactions, again the NSDN comes into play and create many counter-intuitive effects. Like the charge fluctuation between the subsystems is quenched. Also the total charge in the stub or the bubble tends to the same integral value as in the non-interacting case, defying the repulsive interaction between electrons. This is a general feature whenever there is NSDN and does not depend on charge distribution. Because of the quenching of charge fluctuations between the ring and the stub, the persistent currents in the ring can be understood in terms of a ring decoupled from the stub. This can help us understand larger systems for which numerical diagonalization is not possible.

\section{ACKNOWLEDGMENT}

We acknowledge the financial support from the Academy of Finland and from the European Community project ULTRA-1D (NMP4-CT-2003-505457)
[1] M. Buttiker and C. A. Stafford, Phys. Rev. Lett. 76, 495 (1996).

[2] S.Y. Cho, K. Kang, C.K. Kim, and C.M. Ryu, Phys. Rev. B 64, 033314 (2001).

[3] M. Buttiker, Y. Imry and R. Landauer, Phys. Lett. A 96, 365 (1983).

[4] P. Singha Deo, Phys. Rev. B 51, 5441 (1995).

[5] P. Singha Deo, Phys. Rev. B 53, 15447 (1996).

[6] M. Pascaud and G. Montambaux, Phys. Rev. Lett. 82, 4512 (1999)

[7] C.M. Ryu and S.Y. Cho, Phys. Rev. B 58, 3572 (1998).

[8] P. Koskinen and M. Manninen Phys. Rev. B 68, 195304 (2003)

[9] A. Bohr and B. Mottelson, Nuclear Structure (Benjamin, New York) 1975.

[10] M. Koskinen, M. Manninen and S. M. Riemann, Phys. Rev. Lett. 79, 1389 (1997); C. Yannouleas and U. Land- man, Phys. Rev. Lett. 825325 (1999); A. Puente and L. Serra, Phys. Rev. Lett. 833266 (1999); S. M. Reimann and M. Manninen Rev. Mod. Phys. 74, 1283-1342 (2002)

[11] K. Clemenger, Phys. Rev. B 321359 (1985).

[12] P. Singha Deo, P. Koskinen, M. Koskinen and M. Manninen, Europhys. Lett. 63846 (2003); A. Emperador, F. Pederiva, and E. Lipparini Phys. Rev. B 68, 115312 (2003); S. Viefers, P. Koskinen, P. Singha Deo, M. Manninen Physica E 21, 1-35 (2004).

[13] P. A. Sreeram and P. Singha Deo, Physica B 228, 345 (1996).

[14] A. J. Leggett, in Granular Nano-Electronics, Vol. 251 of NATO Advanced Studies Institute, Series B: Physics, edited by D. K. Ferry, J. R. Barker, and C. Jacoboni (Plenum, New York, 1991), p. 297.

[15] E. Tekman and P.F. Bagwell, Phys. Rev. B 48, 2553 (1993). 
[16] M. Koskinen, M. Manninen, B. Mottelson and S. M. Reimann, Phys. Rev. B 63, 205323 (2001).

[17] H. Schomerus, Y. Noat, J. Dalibard, and C. W. J. Beenakker Europhys. Lett. 57, 651 (2002)

[18] M. Born and W. Heisenberg, Z. Phys. 16, 229 (1923).

[19] W. Dybalski and P. Hawrylak, cond-mat/0502161.

\section{Figure Captions}

Fig. 1. (a) A schematic diagram of a ring-stub system. The system is made up of $\mathrm{R}$ sites in the ring and $\mathrm{S}$ sites in the wire or stub. The sites are shown as big dots and the connection between them is shown as lines. Sites in the stub are labeled A and B. (b) A schematic diagram of a ring-bubble system. Sites in the bubble are labeled $\mathrm{A}$ and $\mathrm{B}$.

Fig. 2. Eigen energies of the single particle states of the system in Fig. 1 (a), as a function of $\alpha=2 \pi \phi / \phi_{0}$, in the non-interacting limit of $U=0$ and $V=0$. Here $\phi$ is the total flux through the ring and $\phi_{0}=h c / e$. The ring consists of 9 sites and the stub consists of 2 sites. $E$ represents energy and the unit of energy is $E_{0}=t$.

Fig. 3. Schematic diagrams of a stub (a) and bubble (b) corresponding to Fig. 1, but now with open leads.
Fig.4. The system under consideration is in Fig. 1 (a) with $R=9$ and $S=2$. There are 4 spin up electrons and 4 spin down electrons in the system with $U=2$ and $V=1$. We show here spin up occupation probability $\left\langle n_{A}>\right.$ and $\left\langle n_{B}\right\rangle$ of the two sites $A$ and $B$ in the stub as a function of $\alpha$ where $\alpha=2 \pi \phi / \phi_{0}$. Here $\phi$ is the total flux through the ring and $\phi_{0}=h c / e$.

Fig. 5. Same as in Fig. 4 , but $U=9$ and $V=5 .<n_{A}>$ and $\left\langle n_{B}>\right.$ are individually flux dependent but the sum of them do not depend on flux.

Fig. 6. The first 6 many body levels of a ring with a stub as a function of $\alpha=2 \pi \phi / \phi_{0}$. $\phi$ is the total flux through the ring and $\phi_{0}=h c / e$. Here $R=6, S=2, U=10$ and $V=8$. The unit of energy $E$ is $E_{0}=t$.

Fig. 7. Many body levels of a ring with a potential impurity as a function of $\alpha=2 \pi \phi / \phi_{0} . \quad \phi$ is the total flux through the ring and $\phi_{0}=h c / e$. Here $R=6, S=0$, $U=10$ and $V=8$. One of the sites has a site energy of $5 t$. The unit of energy $E$ is $E_{0}=t$. 


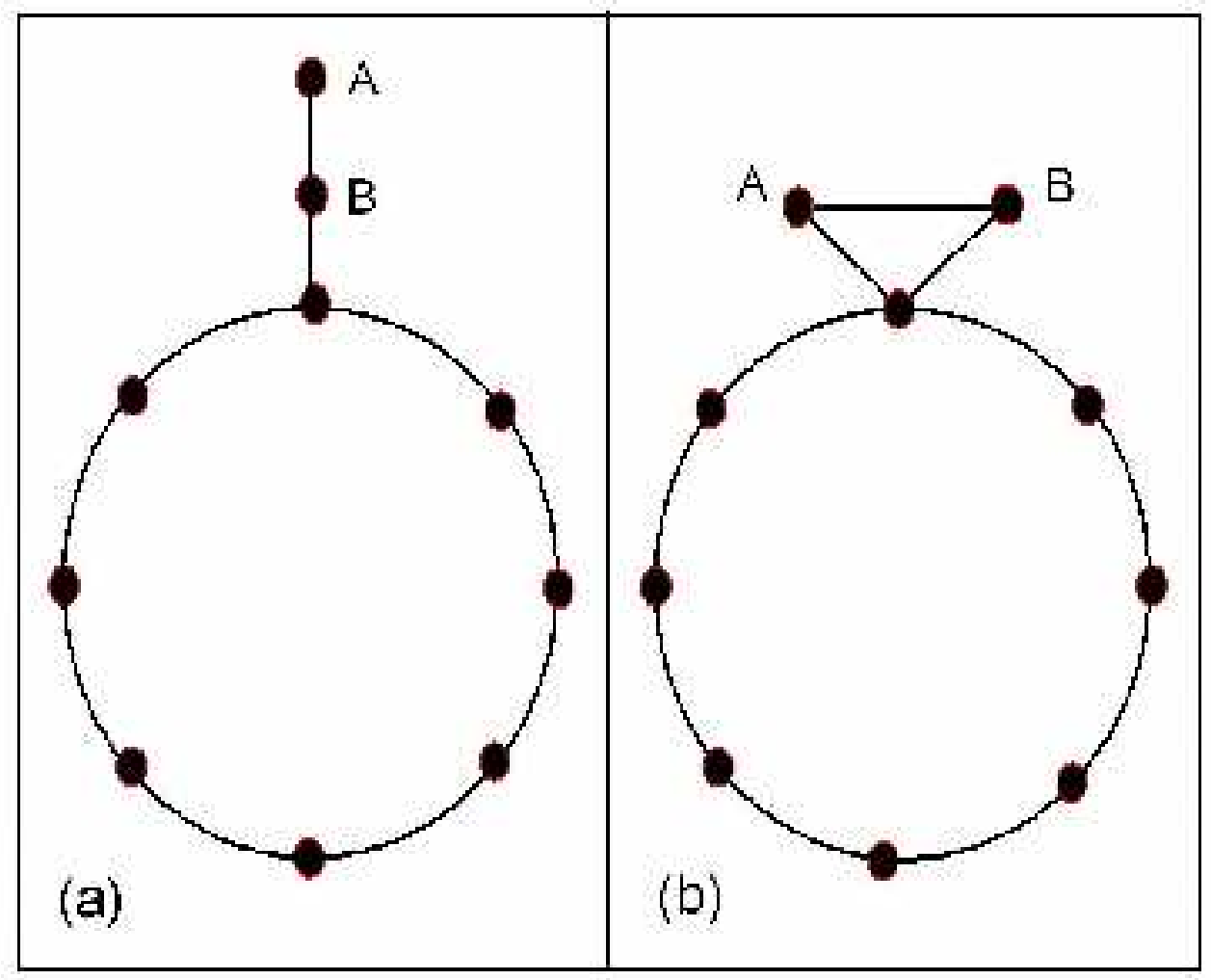




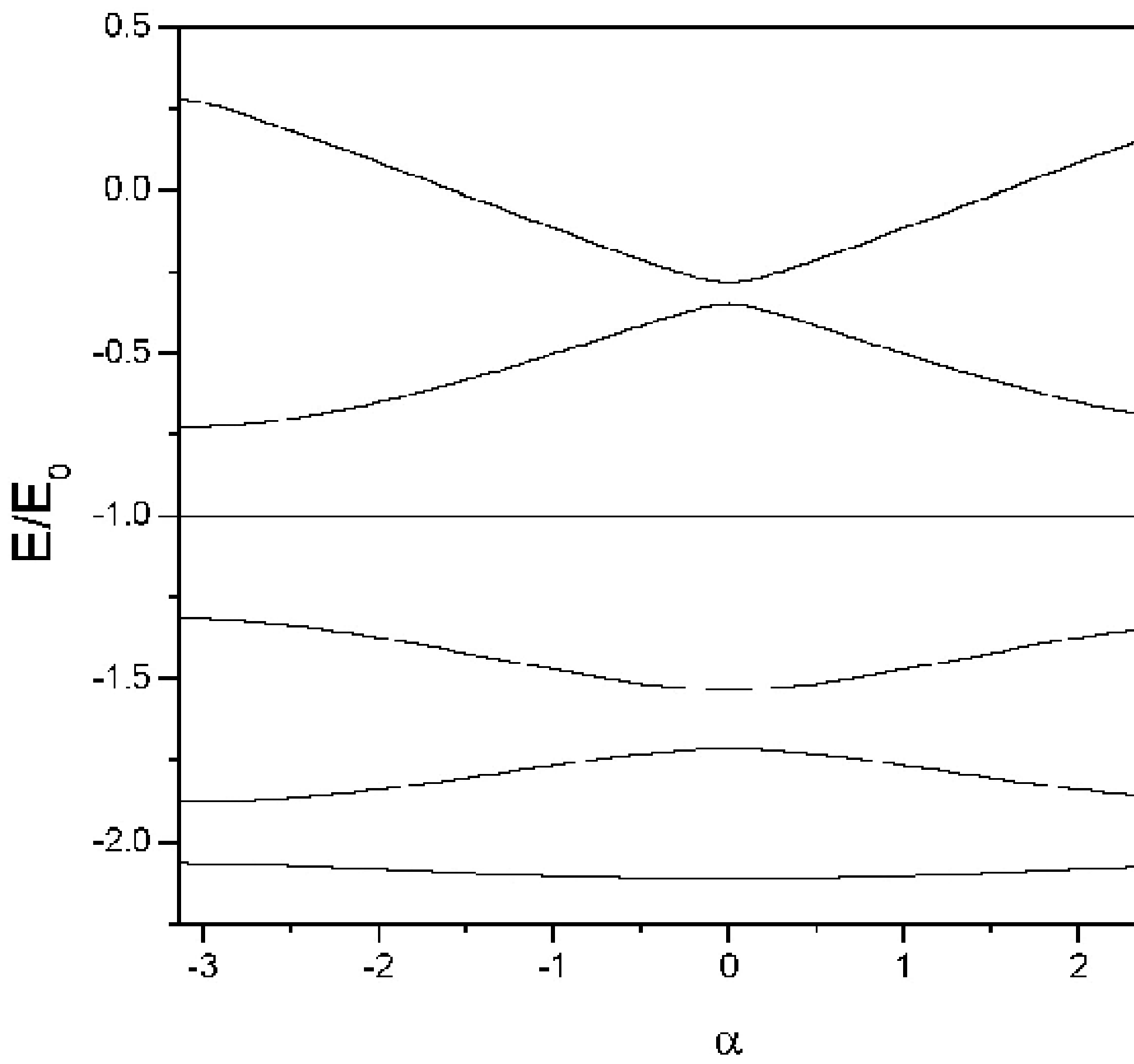




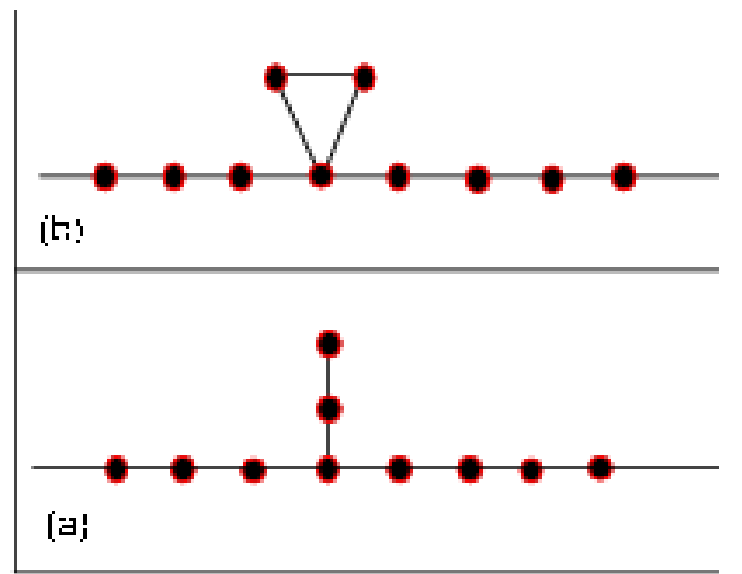




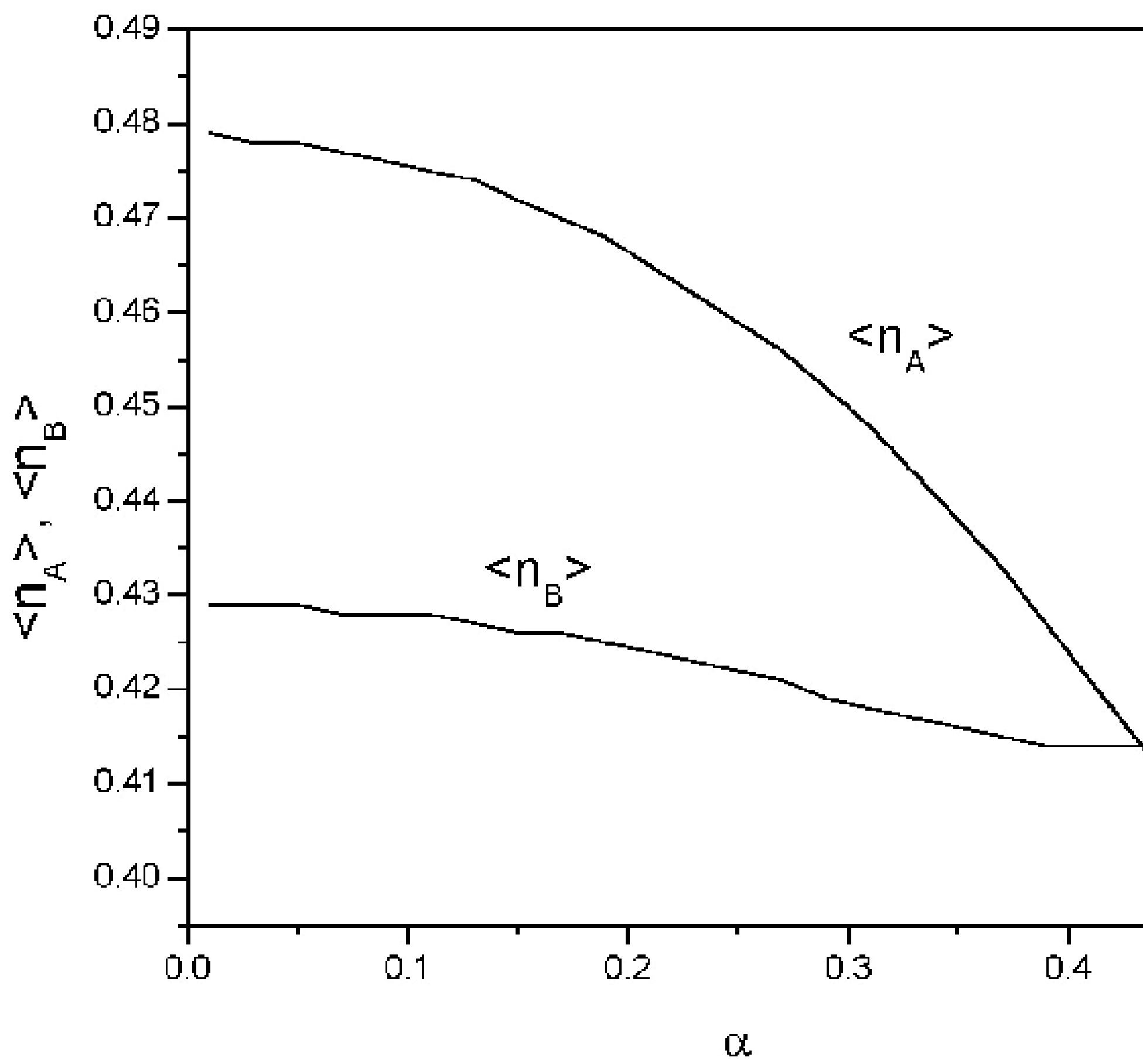




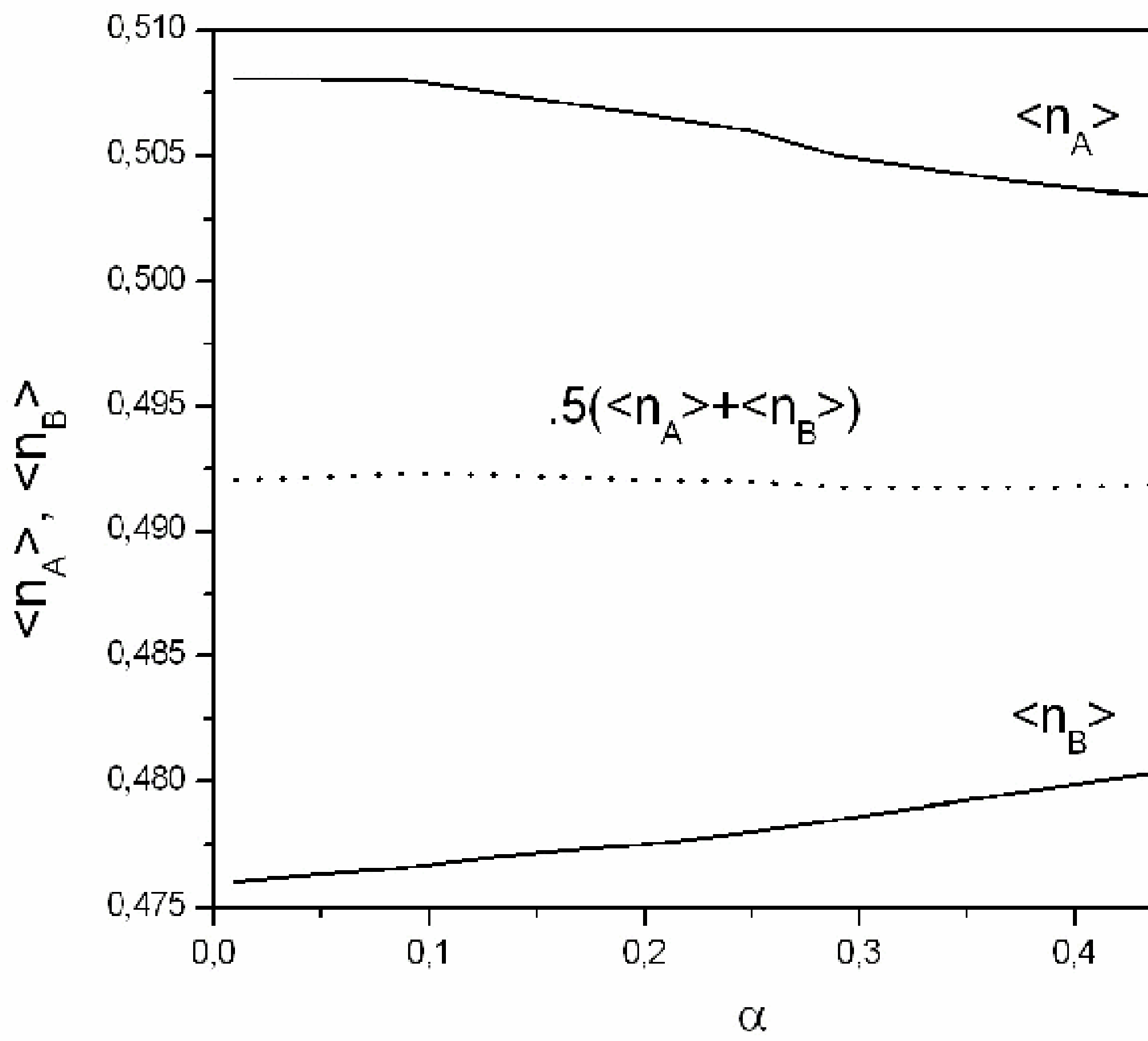




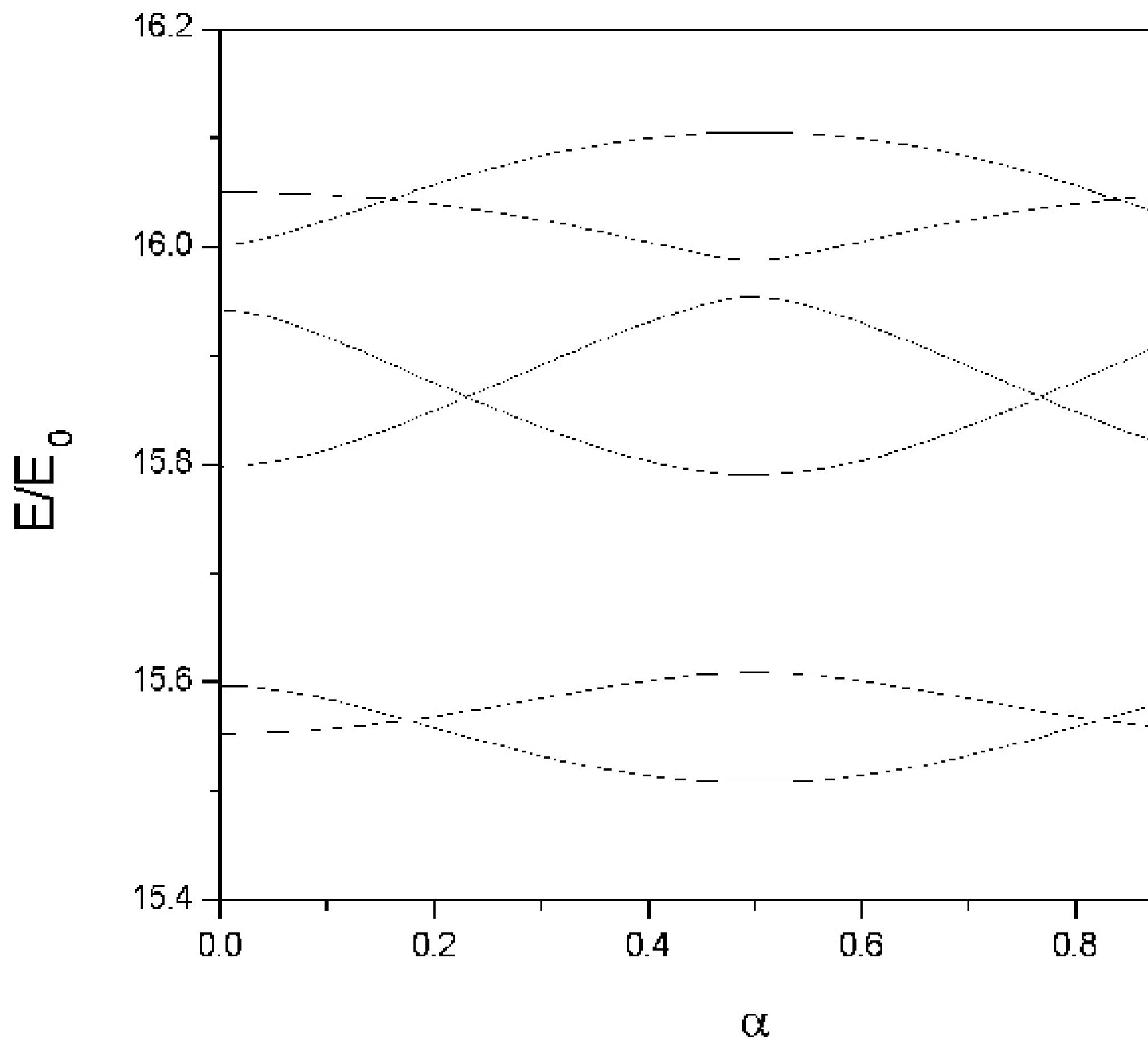




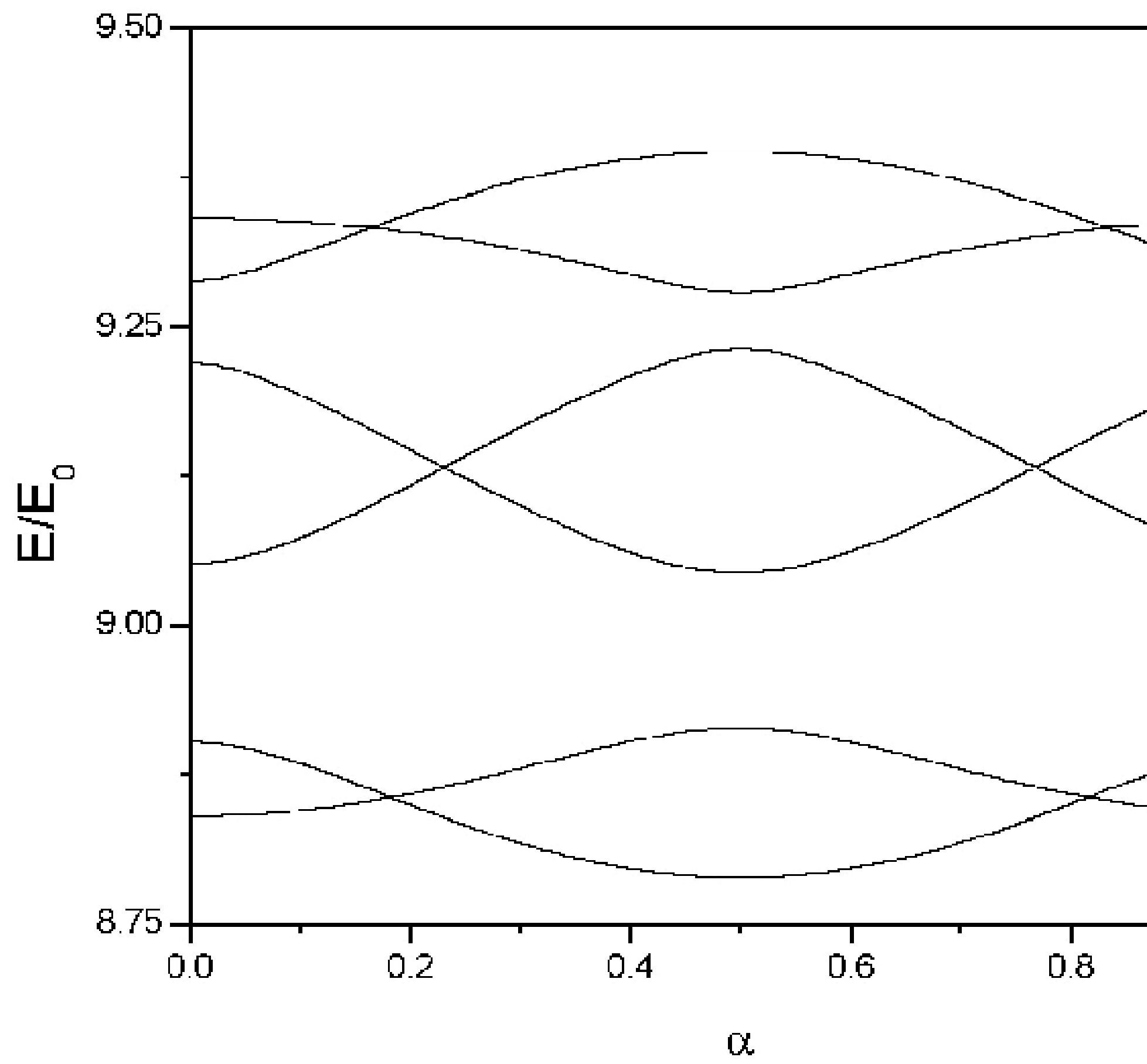

